\title{
Research article \\ Study on the arthroscopic repair of the rotator cuff tear
}

\author{
Mihai Tudor Gavrilă ${ }^{1}$, Cristea Stefan ${ }^{1}$, Emanuel Antoneac ${ }^{1}$ \\ ${ }^{1}$ Carol Davila University of Medicine and Pharmacy, Bucharest, Romania \\ ${ }^{2}$ St. Pantelimon Hospital, Department of Orthopedics and Traumatology, Bucharest, Romania
}

\begin{abstract}
Rotator cuff tear (RCT) is common shoulder pathology. The majority of the patients do not require surgical treatment, their evolution being favorable under conservative treatment. In other cases, the symptomatology does not improve without surgery. This can be open or less invasive, i.e. arthroscopic.

Material and Methods: We evaluated 30 cases of rotator cuff (supraspinatus) tear, operated on arthroscopically between 2015 and 2017. All surgeries were done arthroscopically, by the same surgeon. The data were collected using the Constant score and the SST score, calculated preoperatively and postoperatively at 12 months. The average age was $\mathbf{5 2 . 6}$ for female patients and 53.2 for male patients.

Results: they improved after treatment. The constant score increased from 44 to 84 and SST from $25 \%$ to $83.3 \%$. All patients were treated closing the defect, using one or more anchors, with a simple or a double-row technique. Conclusions: The evolution was good with significant improvement in terms of pain and strength.
\end{abstract}

\footnotetext{
Keywords

: rotator cuff tear, arthroscopy, shoulder, repair

Highlights

$\checkmark$ Rotator cuff tear is a pathology often meet in adult population, especially on male patients. In majority of cases treatment is conservative but, for subjects who don't respond at this form of treatment surgery is required.

$\checkmark$ Open or arthroscopic surgical procedures proved to have relative similar results on patients with rotator cuff tear in term of pain relief and range of motion.
}

To cite this article: Gavrilă MT, Cristea S, Antoneac E. Rotator cuff tear arthroscopically repair study. J Clin Invest Surg. 2019; 4(1): 38-41. DOI: 10.25083/2559.5555/4.1/38.41 


\section{Introduction}

The shoulder is the most mobile joint in the body. Its mobility is the consequence of the asymmetry between glenoid and proximal humerus. To stabilize the glenohumeral joint, a series of structures are involved: capsula, ligaments and muscles. The rotator cuff is an assembly of four muscles that rotate the shoulder (Figure 1).

The subscapularis is situated in front of the joint, helping us rotate the arm posteriorly. The infraspinatus and teres minor rotate the arm externally and the supraspinatus, which is situated superiorly, allows us to lift arm above the head.

The high mobility of this joint exposes it to different trauma, producing a variety of lesions (from the simple irritation of the tendon to its complete rupture). In the majority of cases, the cause of the tear is direct fall on the shoulder, or the repetitive lifting of arm above the head, as we see in sports (swimming, tennis, basketball, volley, etc.) or in household activities (painting, plastering, etc.).

In the last cases, the repetitive movements produce an overuse of the tendon with rupture of tissue in different degrees. The most exposed muscle is by far the supraspinatus, because it is situated between two bone structures: the acromion above and the humeral head below.

\section{Materials and Methods}

Some studies show that the dominant hand and the age are two factors involved in RCT. The dominant hand is utilized more and, because of that, more stress is put on it. With age, vascularization at the level of tendon decreases, this being another factor that increases the risk of tear.

Other factors such as diabetes mellitus, hypertension or smoking could be associated with RCT, but studies are not very conclusive. In the literature, there is no difference between males and females regarding the occurrence of RCT.

In our study, 30 patients were included: both $\mathrm{M}$ and $\mathrm{F}$. Average: with rotator cuff tear. The tears were classified using DeOrio and Cofield system (5): $1 \mathrm{~cm}, 1-3 \mathrm{~cm}, 3-5 \mathrm{~cm}$ and greater than $5 \mathrm{~cm}$ gap in the mass of the tendon which means small, medium, large and massive tears. In our cases, the selected patients had small, medium and large tears. The massive tear could not be repaired. All of them were operated on arthroscopically by the same senior surgeon. None of them underwent performance sports activity. Some of them were smokers; others had diabetes mellitus or hypertension.

The symptomatology in these patents consisted in pain which was mild or severe, predominately during the night or during movement with the progressive loss of the capacity to lift the arm. A series of tests were suggestive for this pathology. Neer test, Hawkins test, Jobe test and abduction arch test were all positive. Patients note the onset of the symptoms during a traumatic event and the symptomatology becomes worse with daily activities.

The imaging investigation confirms the diagnosis. The $\mathrm{X}$-ray reveals an acromion Bigliani type III, the superior ascension of humeral head in the total rupture of the rotator cuff. The most sensitive investigations are MRI and ultrasounds, both revealing the defect in the muscle mass of the rotator cuff (Figure 2).

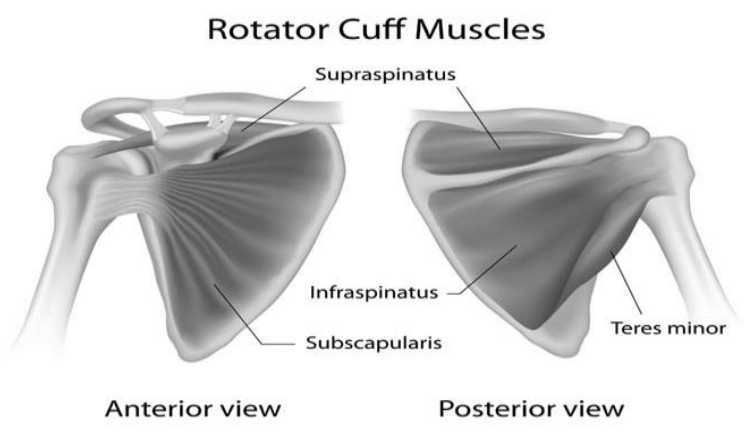

Figure 1. Rotator cuff muscles
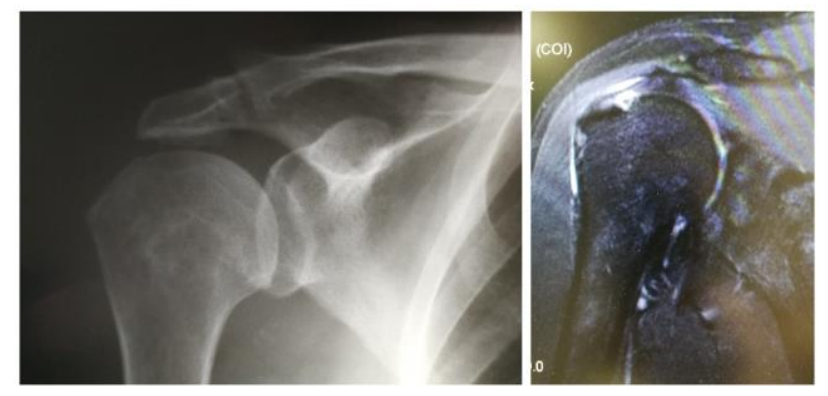

Figure 2.

Left- Radiologic aspect of Bigliani III acromion;

Right- MRI revealing the cuff tear (personal archive)

Before surgery, a form was filled in (SST and Constant score). The same forms were completed a year after surgery.

Surgery was done in beach-chair position (Figure 3) under general or loco-regional anesthesia. We used threefour portals: posterior, anterior and two laterals (2-3).

At the beginning, a diagnostic gleno-humeral visualization was performed, followed by subacromial decompression: bursectomy, acromioplasty (whenever necessary) and the resection of the coracoacromial ligament. Subsequently, the torn tendon was debrided, and the footprint prepared with the burr.

The repair was done using a single or a double-row technique (one, two or more anchors: cork-screw and PushLock) (1). For the single-row technique, the anchors 
were placed in the cuff footprint. With the suture passed through the tendon, a knot was done.

For the double-row technique, the first anchor (or anchors) was (were) placed in the medial side of the footprint. Sutures were passed through the tendon tissue at $5-10 \mathrm{~mm}$ away from the edge of the tear and tied in horizontal mattress fashion. The sutured arms were then introduced in the second anchor (anchors) that was (were) placed laterally, perpendicular on the cortical surface of the humerus, $5-10 \mathrm{~mm}$ distal to the lateral edge of the greater tuberosity.

Postoperatively, the suture was protected with a special device, an abduction-pillow (with forearm in the neutral position and the affected arm at 30 degrees of abduction). Routine follow-up visits occurred at 3 days, 2 weeks, 6 weeks, 3, 6 and 12 months postoperatively. The sutures were removed after 2 weeks. During the first 4-6 weeks, the shoulder was immobilized in an abduction-pillow.

The active range of motion of the wrist and elbow was allowed, but only passive shoulder range of motion was permitted. At 6 weeks postoperatively, the active range of motion started and at 3 months, active resistance musclestrengthening exercises were begun. At 6 months, full normal activities were allowed.

The function of the shoulder function was measured before surgery and 1 year postoperatively. We used two international scales to evaluate the clinical outcomes: the simple shoulder test (SST) and the Constant test.

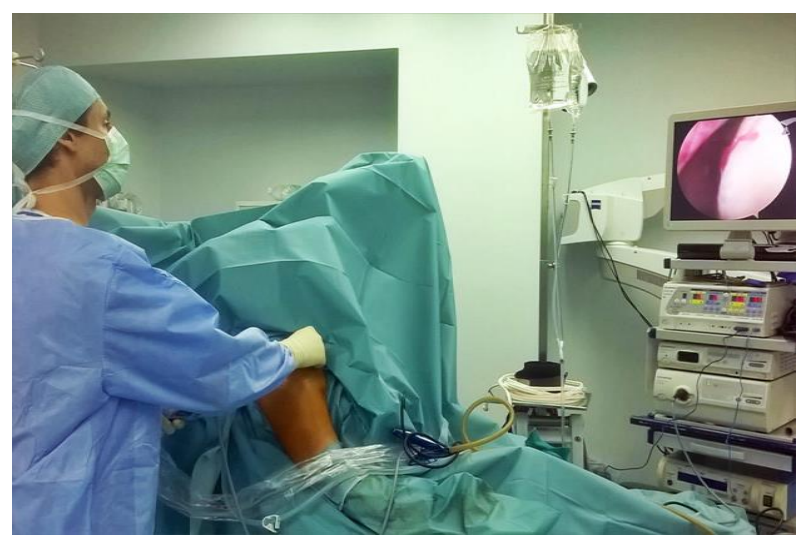

Figure 3. Beach-chair position in arthroscopic surgery

\section{Results}

From the total of 30 patients operated on arthroscopically for RCT, 24 were male and 8 were female patients whose age ranged between 42 and 72; the average age was 52.6 for females and 53.2 for males. The majority of the patients were male. 17 cases were repaired using a single-row technique and 13 using a double-row technique.
All surgeries were done arthroscopically by the same doctor, in the beach-chair position. The repair of the torn tendon was done using one, two or more anchors.

Postoperatively, every patient protected the suture with abduction-pillow for 4-6 weeks. The healing process lasted for several months to one year. The active range of motion was allowed at 6 weeks postoperatively.

The clinical data were evaluated using the Constant score and the SST score preoperatively and one year after surgery by the attending physician. The active range of motion was calculated with a goniometer at the site of pain.

The follow-up occurred at 2 weeks, 6 weeks and then at 3, 6 and 12 months. We found an improvement in the Constant score from 44 to 84 and for the SST from $25 \%$ to $83.3 \%$.

No intraoperative or postoperative complications were observed. None of the patients had nerve or vessel damage, suture anchor problems or wound-site infection.

Our operative goal was to relieve pain. All patients experienced improvement in terms of pain and mobility.

\section{Discussions}

The Gleno-humeral joint is the most mobile joint in the body, due to the incongruence between bone segments. The stability of the joint is maintained by the capsuloligamentous structures and muscles that surround the humeral head.

All these four muscles are attached to the bone in a specific aria, creating the so-called footprint of the muscle (around $1 \mathrm{~cm}$ in diameter).

The main stabilizers of the joint are the four muscles that create the rotator cuff. These are: the subscapularis, the supraspinatus, the infraspinatus and the teres minor. Overhead activities can produce damage at the level of these muscles.

The rotator cuff tear is a common pathology of the shoulder. It is known that both the dominant hand and age are involved in the occurrence of the tear at this level. In many cases, the conservative treatment proved to be helpful with pain relief and regaining the range of motion. It consists of physiotherapy and administration of NSAIDs.

When symptomatology is resistant, the surgical treatment must be undergone. It is known that half of all tears enlarge over time and become more painful.

One option is open surgery, but nowadays, with the development of technology, arthroscopic intervention proved to have similar results. It demands a skillful surgeon, but also a well-informed patient. This kind of surgery is characterized by less morbidity than open surgery. 
We treated 30 patients, the majority of them male. In our practice, males developed more RTCs, probably because they are more involved in physical activities than females. The procedure was performed by the same surgeon arthroscopically, closing the defect with the help of anchors. The goal was to restore the footprint of the torn tendon, especially the supraspinatus muscle.

The goal of RTC surgery is first to relieve pain and second to improve the motion and the functionality of the shoulder. The healing time was long (months) and required the collaboration with the patient along the process.

An intensive physiotherapy program was followed by each patient to restore a range of motions and regain the strength of the rotator muscles.

Preoperatively and postoperatively (one year after surgery), specific scores were measured to evaluate the results of the treatment. The constant score increased from 44 to 84 and the SST score increased from $25 \%$ to $83.3 \%$.

The results proved the efficiency of arthroscopic surgery in the treatment of RCT (4-6) with low complications and a maximum of benefits in terms of pain relief and regaining motion and strength (7-8).

Because of the benefits, this kind of surgery will be the future treatment in shoulder muscle pathology.

\section{Conclusions}

The rotator cuff muscles are very important in the mobility and stability of the joint. With age, especially for the dominant hand, damage will occur at this level.

The rotator cuff tear is a pathology often seen in the adult population, especially in males. It is the result of repetitive trauma at the level of the shoulder.

The diagnosis is supported by the clinical examination, the patient's history and the imaging investigations (MRI, $\mathrm{Rx}$, shoulder scans).

Sometimes RTC is symptomatic and in the majority of cases, the treatment is conservative. For patients who do not respond to this form of treatment, surgery is the best option.

Surgery can be open or arthroscopic, both having almost similar results in terms of pain relief and range of motion.

Arthroscopic surgery provided satisfactory clinical results in the patients with rotator cuff tear, resistant to conservative treatment.

It allows improvement in terms of pain and mobility with low morbidities. It requires a skillful surgeon, trained in this type of surgery. The cosmetic and functional results make arthroscopy the treatment of choice of this pathology.

\section{Conflict of interest disclosure}

There are no known conflicts of interest in the publication of this article. The manuscript was read and approved by all authors.

\section{Compliance with ethical standards}

Any aspect of the work covered in this manuscript has been conducted with the ethical approval of all relevant bodies and that such approvals are acknowledged within the manuscript.

\section{References}

1. Aydin N, Kocaoglu B, Guven O. Single-row versus double-row arthroscopic rotator cuff repair in small to medium -sized tears. J Shoulder Elbow Surg. 2010; 19(5): 722-5. PMID: 20303287, DOI: 10.1016/j.jse.2009.11.053

2. Burkhard SS. A stepwise approach to arthroscopic rotator cuff repair based on biomechanical principles. Arthroscopy. 2000; 16(1): 82-90. PMID: 10627351

3. Burkhard SS, lo IKY, Brady PC. Burkhart's View of the Shoulder: A cowboy's guide to advanced shoulder arthroscopy 2006, 1s ed. Philadelphia: Lippincott Williams and Wilkins. ISBN-13: 978-0781780001

4. Constant CR, Murley AH. A clinical method of functional assessment of the shoulder. Clin Orthop relat Res. 1987; (214): 160-4.

5. DeOrio JK, Cofield RH. Results of a second attempt at surgical repair of a failed initial rotator-cuff repair. $J$ Bone Joint Surg Am. 1984; 66(4): 563-7. PMID: 6707035

6. Lafosse L, Brozska R, Toussant B, Gobezie R. The outcome and structural integrity of arthroscopic cuff repair with use of the double row suture anchor technique. J Bone Joint Surg Am. 2007; 89(7): 153341. PMID: 17606793, DOI: 10.2106/JBJS.F.00305

7. Lorbach O, Wilmes $\mathrm{P}$, Brogard P, Seil R. Complications related to implants in arthroscopic shoulder surgery. Orthopade. 2008: 37(11): 1073-9. PMID: 18813907, DOI: 10.1007/s00132-008-1347-9.

8. Weber SC, Abrams JS, Nottage WM. Complications associated with arthroscopic shoulder surgery. Arthroscopy. 2002; 18 (2 suppl 1): 88-95. PMID: 11828349 